\title{
SUR QUELQUES COPÉPODES \\ PARASITES PROVENANT DE LA RUSSIE \\ SOUS-CARPATHIQUE (TCHÉCOSLOVAQUIE)
}

PAR

Th. MONOD

(Paris) et

\author{
V. VLADYKOV
}

(Toronto)

\section{INTRODUCtion}

Au cours de ses recherches sur la faune ichthyologique de la Russie sous-carpathique(1), l'un d'entre nous s'est préoccupé de réunir quelques matériaux parasitologiques. Les collections recueillies intéressent divers groupes zoologiques et leur inventaire sera publié au fur et à mesure de leur détermination. L'extrême rareté des travaux portant sur la faune d'eau douce de cette région de la république tchécoslovaque confère à ces observations un intérêt particulier. $\Lambda$ joutons enfin que l'éthologie des espèces a spécialement retenu l'attention du collecteur.

Nous nous bornerons ici à l'étude des Copépodes, représentés par 5 espèces, appartenant à 4 genres et 2 familles.

\section{INVENTAIRE DE LA COLLECTION}

Liste des espèces recueillies

La collection renferme les espèces suivantes :

Ergasilidæ.

1. Ergasilus sieboldi von Nordmann, 1832.

Lernæopodidæ.

2. Achteres percarum von Nordmann, 1832.

3. Tracheliastes polycolpus von Nordmann, 1832.

4. Tracheliastes stellatus (Mayor, 1824).

5. Basanistes huchonis (Schrank).

(1) V. Vladyoov, Ryby podkarpatské rusi a hlavni způsoby rybolovu, 147 p., 75 fig., 1 carte, Užhorod 1926. - Du même : Lès poissons de la Russie sous-carpathique (Mém. Soc. Zool. France, 1931). Sous presse.

Annales de Parasitologie, t. IX, $\mathrm{n}^{\circ} 3 .-1^{\text {er }}$ mai 1931 , p. 202-224. 
Nomenclature détaillée des échantillons

Ergasilidæ

\section{Ergasilus sieboldi von Nordmann, 1832}

Ergasilus sieboldi von Nordmann, 1832, p. 15, pl. 2, fig. 1-9. Kröyer, 1863, p. 237, pl. 13, fig. $2 a-b$. - Claus, 1875, p. 339, pl. 23, fig. 12-18. - Gadd, 1904, p. 4, pl. 1, fig. 20-25. - Neresheimer 1909 a, p. 72-73, fig. 312, 314. - Pesta, 1928, p. 129, fig. 108.

Localité. - 5 , sur les lames branchiales d'un Gobio gobio carpathicus Vladykov, M. Berezna, riv. Ouge [Uż], 4-VII-1926.

Distribution. - Toute l'Europe.

Remarques. - C'est la forme européenne banale, ou du moins admise jusqu'ici comme telle. On a décrit d'Europe plusieurs autres espèces d'eau douce, E. gibbus von Nordmann, 1832, E. trisetaceus von Nordmann, 1832, E. surbecki Baumann, 1913, E. hoferi Borodine, 1915, distinguées principalement par la chétotaxie des branches furcales, laquelle pourrait, d'après certains auteurs, présenter une certaine variabilité (Wegener 1910, Zandt, 1924, p. 258). Enfin Messjatzeff a récemment décrit un $E$. baicalensis (1926, p. 122-125, fig. 1-5).

Observations éthologiques. - Forme paraissant peu commune dans la région envisagée.

\section{Achteres percarum von Nordmann, 1832}

Achteres percarum von Nordmann, 1832, p. 63 , pl. 4, fig. 1-11, pl. 5, fig. 1-7. - Claus, 1862, p. 287, pl. 23-24. - Neresheimer, $1909 a$, p. 78 , fig. $329-330$. - Scott, 1913, p. 193-194, pl. L, fig. 6-8, LIX, fig. 7-8, LXIV, fig. 1-3. — Wilson, 1915, p. 629-631, pl. 36, fig. 96-97.

Localités. - a. 5 \%, sur Lucioperca volgensis (Gmelin) (2 à la face interne de l'opercule, 2 sur les lames branchiales, 1 sur la langue), Tchop [Čp, Czop], riv. Tisa.

b. 1 , sur Lucioperca volgensis (Gmelin), sur le premier arc branchial, environs de Oujorod [Užhorod], riv. Ouge Už], 25-II-1927.

c. 5 Q , sur Perca fluvialilis vulgaris Schaeffer, dans la cavité buccale et sur les branchies (exemplaires bleus), Tchop Č́op, Czop], riv. Tisa.

Distribution. - Toute l'Europe ; une forme affine en Sibérie, A. sibirica Messjatzeff (1926, p. 130-133, fig. 16-19). 
Remarques. - Les épines unciformes des deux branches de l'antenne sont recourbées vers l'extérieur et non vers l'intérieur, comme le représente von Nordmann (1832, pl. 5 , fig. 6 n-o).

Observations éthologiques. - Relativement fréquent sur les Percidés cités plus haut, habitant l'un et l'autre le cours inférieur et la partie inférieure du cours moyen des rivières, inconnu par contre sur les représentants des genres Aspro et Acerina qui habitent des eaux à courant plus rapide.
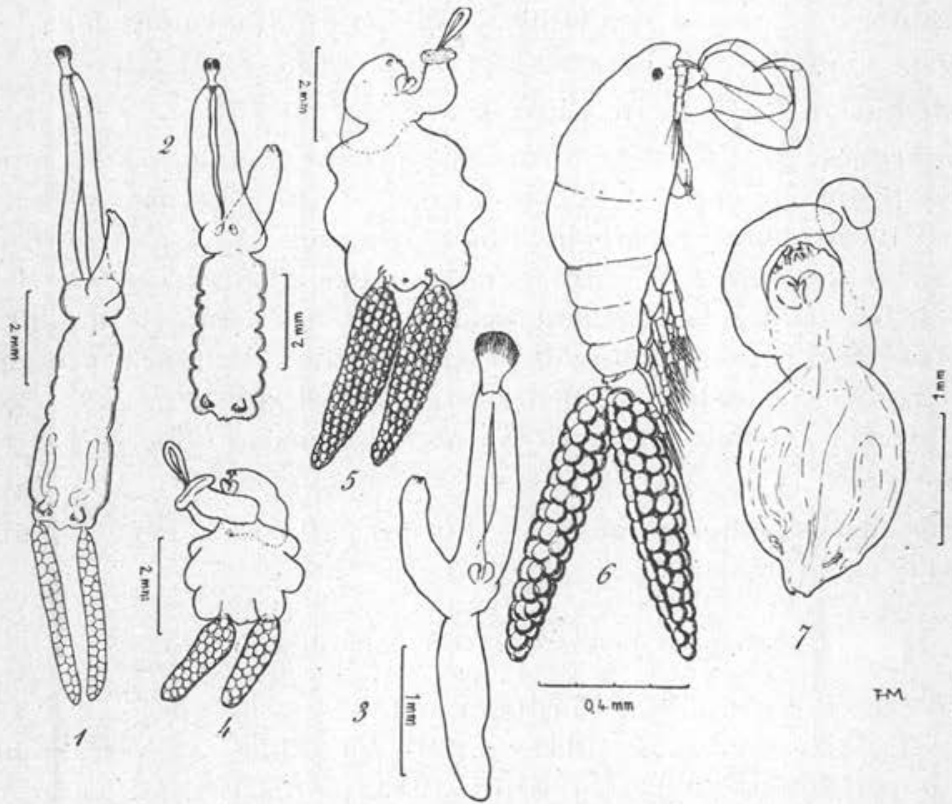

Fig. 1. - 1. Tracheliastes polycolpus von Nordmann $ᄋ$ (a) (1), -2 . Id. (b). - 3. Id . juv. (d). - 4. Basanistes huchonis (Schrank) 우 (e). - 5. Id. (c). - 6. Ergasilus sieboldi von Nordmann $ᄋ$ (sur Esox lucius L., Secon, Oberbayern, Dt. Karl Hofeneder, 8.7. 1929). -7 . Achteres percarum von Nordmann $Q$ (b).

\section{Tracheliastes polycolpus von Nordmann, 1832}

Tracheliastes polycolpus von Nordmann, 1832, p. 95-99, pl. 7 . - Kollar, 1836, p. 85-86. - Vejdovsky, 1877, p. 15-46, pl. 2-4. Kunz, 1877, passim et pl. 26, fig. 33 et 27 , fig. 42 . - Neresheimer, $1909 a$, p. 79, fig. 333. - Messjatzeff, 1926, p. 133-134, fig. 20-23.

(1) Les lettres en italiques se rapportent aux provenances (hôtes et localités), dans l'inventaire systématique de la collection. 
Localités. - a. 4 \&, sur Abramis brama (L.), are branchial, Oujorod [Užhorod], riv. Ouge [Už], 1927.

b. 1 ㅇ, sur Barbus meridionalis petenyi Heckel, Oujorod [Užhorod], riv. Ouge [Ǔ̃], 1925.

c. 5 (dont 2 juv.), sur Barbus barbus (L.), base de la dorsale [Užhorod], riv. Ouge [Ǔ̌], 1925.

d. 1 q, juv., sur Barbus barbus (L.), anale, Neresnitze [Neresnice], riv. Teresva (affl. de Tisa), 1925.

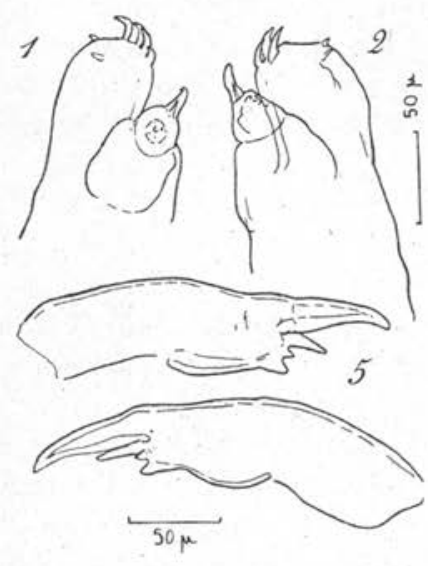

FM.

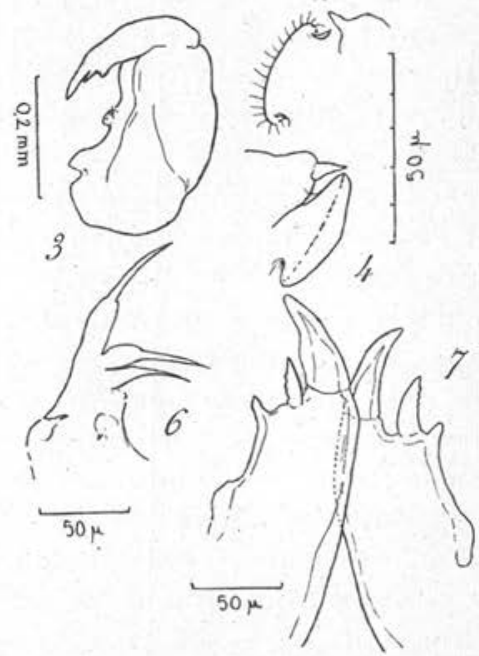

Fig. 2. - Achteres percarum von Nordmann $ᄋ$ (1-6: b, $7: c)$. - 1-2. Antennes. 3. Maxillipèdes. - 4. Organe marginal interne des deux maxillipèdes. - 5 . Extrémité distale des deux maxillipèdes. - 6. Maxillule. - 7. Extrémité distale des deux maxillipèdes $(c)$.

e. 4 f, sur Barbus barbus (L.), parties proximales des ventrales et pectorales, Neresnitze [Neresnice], riv. Teresva (affl. de Tisa), 12-VIII-1924.

Distribution. - Europe centrale et orientale, Sibérie.

Remarques. - Nous rapportons les échantillons examinés à T. polycolpus, tel qu'il est décrit et figuré par von Nordmann et Vejdovsky.

Von Nordmann (1832, pl. 7, fig. $3 b-d$ ) et Vejdovsky (1877, pl. 3, fig. 1-2) figurent des antennes qui ne sont pas tout à fait semblables. Von Nordmann (1832, p. 96), écrit : "An ihrem Ende sind sie in zwei Forsätze gespalten, einen nach innen gerichteten, welcher dick und ausser einigen Borsten noch mit einer grösseren 
Spitze versehen, und einem aüsseren, dem ein grosser Haken so eingegliedert ist, dass er beim Umbiegen in den $Z$ wischenraum zweien kleineren Spitzen hineinpasst. "

Vejdovsky $(1877$, p. 18-22) insiste sur le fait que les antennes et les maxillipèdes de ses spécimens (des environs de Prague) ne sont pas tout à fait semblables aux appendices figurés et décrits par von Nordmann, et, pour marquer les divergences reconnues entre la description de von Nordmann et ses échantillons, il crée pour ces derniers la variété phoxini $(1877$, p. 19).

Les antennes de nos spécimens concordent à peu près avec la description de von Nordmann; cependant, la rame interne est notablement plus volumineuse que sur la figure du descripteur, sa dent interne est souvent invisible ou difficile à apercevoir (1) et son extrémité, largement arrondie, ne porte pas d'épines saillantes (chez l'adulte), mais simplement un bord "granulé ", ou mieux, finement ponctué.

Vejdovsky figure une antenne à rame interne courte (n'ayant nullement, comme chez les échantillons sub-carpathiques, la forme d'un vaste lobe claviforme surplombant la griffe de la rame externe) et terminée par deux petites épines. Il n'y a pas de raison de suspecter l'exactitude de ces renseignements (en attendant l'indispensable examen de topotypes), et il faut admettre l'existence de plusieurs types d'antennes dans l'intérieur de l'espèce ; nous y reviendrons plus bas.

Les maxillipèdes, eux aussi, auraient chez T. polycolpus phoxini, d'après Vejdovsky, une forme un peu différente de celle qu'ils ont chez la forme typique, que l'auteur tchèque ne connaissait sans doute que par la description de von Nordmann. Les différences que nous avons observées nous-mêmes entre les maxillipèdes d'exemplaires d'âges variés, et - il faut le dire - la difficulté que l'on éprouve à caractériser, même par le dessin, la forme de ces appendices réduits, dégénérés, sans contours bien définis, obligent à considérer comme très délicat l'emploi des maxillipèdes pour l'établissement de coupures taxonomiques.

Nos exemplaires nous ont présenté des maxillipèdes de deux types bien différents. Chez les jeunes $(1,5 \mathrm{~mm}$.), le maxillipède n'est pas sans rapport avec celui figuré par Vejdovsky $(1877$, pl. 2, fig. $1 b$ et 3 ) ; il est cylindroïde, avec l'indication de plusieurs articles, et surmonté d'une griffe robuste fortement recourbée qui s'applique sur la face latérale de l'article précédent, le long d'une

(1) Vejdovsky spécifie n'y être jamais parvenu (1877, p. 22). 
saillie digitiforme de l'angle distal-interne de cet article. Chez l'adulte $(7 \mathrm{~mm}$.) le maxillipède est constitué, à ce qu'il semble, par une partie piriforme, dilatée, rétrécie à son extrémité distale que surmonte une griffe très petite; on distingue, au bord interne de

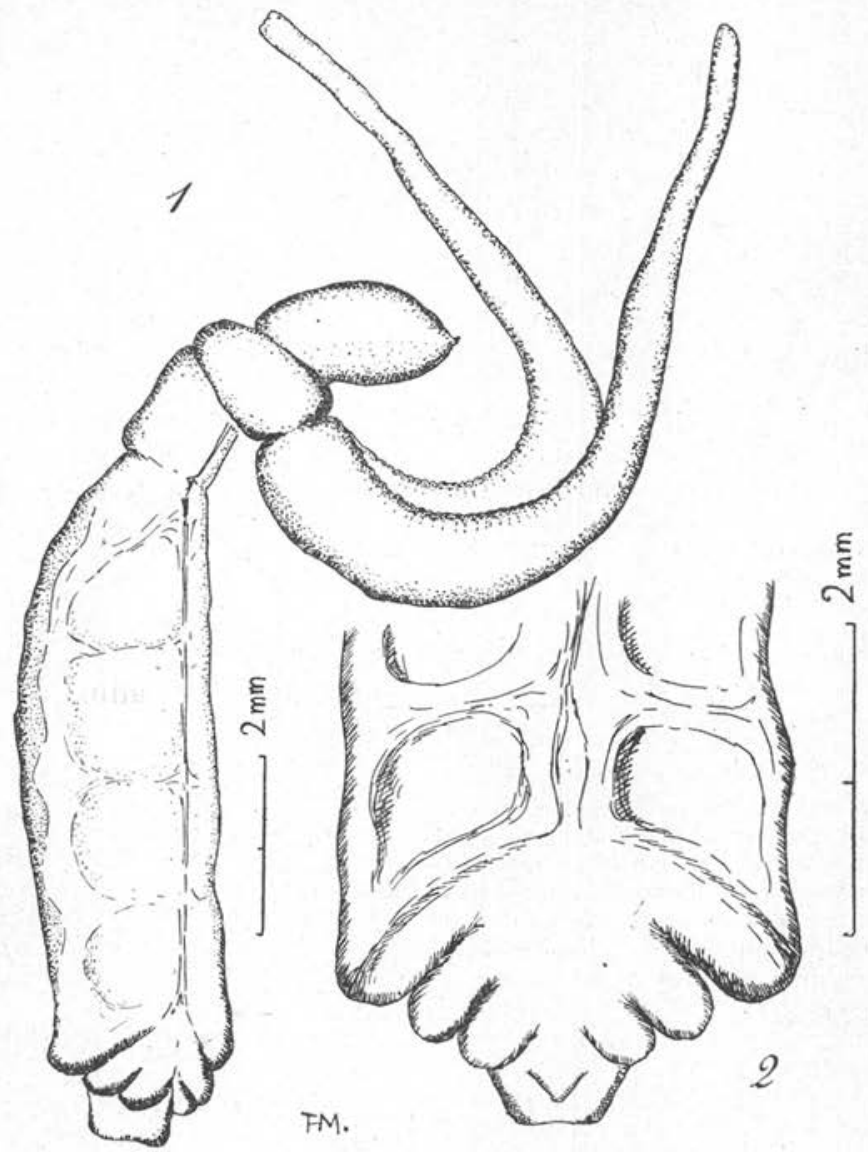

FIG. 3. - Tracheliasles stellalus (Mayor) $\bigcirc(a)$. - 1. Aspect latéral. 2. Extrémité anale du corps, en vue ventrale.

l'article proximal, un orifice auquel aboutit un canal et l'intérieur de l'article paraît rempli par une glande, correspondant vraisemblablement à celle figurée par Vejdovsky (1877, pl. 2, fig. $3 d-z)$.

Observations éthologiques. - L'espèce est signalée comme fixée sur les nageoires des Cyprinidés : nos exemplaires des branchies d'Abramis brama ne sont pas distincts de ceux des nageoires. Ce 
parasite paraît particulièrement fréquent sur des formes d'eau plutôt courante (Barbus, Chondrostoma, Phoxinus) et rare sur les formes d'eau stagnante ou peu courante (Leuciscus idus, Abramis brama).

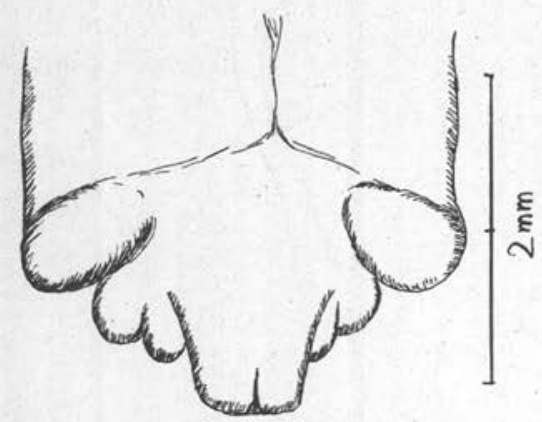

Fig. 4. - Tracheliastes stellatus (Mayor) 우 (a). - Aspect dorsal de l'extremité anale du corps.

Fig. 5. - Tracheliastes stellatus (Mayor) (b). - 1. As pect ventral (I-IV : segmentation du " tronc "; $a$, petit organe dorsal, pair ; $b-g$, invaginations ventrales, disposées par paires, et dont le cul-de-sac, rempll de corps étrangers, apparaît de teinte foncée). - 2. L'un des $\alpha$ bras $»$.

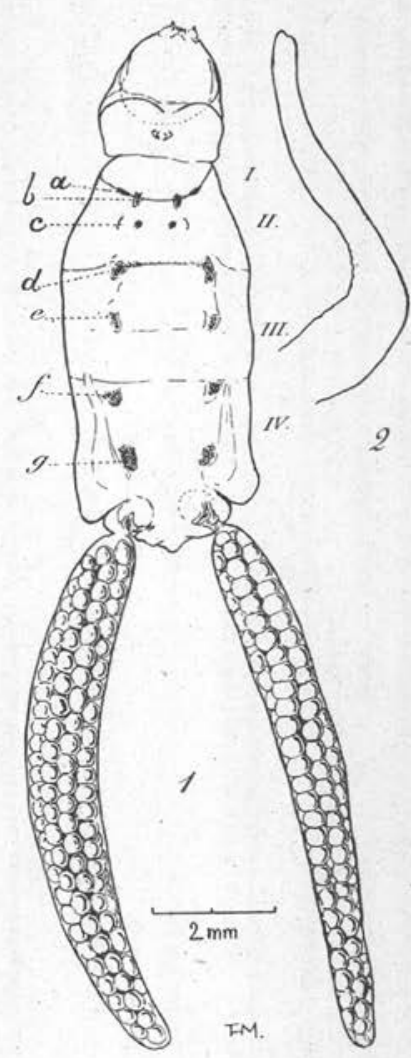

\section{Tracheliastes stellatus (Mayor, 1824)}

Lerneopoda stellata Mayor, 1824, p. 24, pl. 1, fig. 1-5. L. [erneopoda] stellata Desmarest, 1825, p. 351. - Lernæopoda stellata Rathke, 1835, p. 155 et passim. - Tracheliastes stellifer Kollar, 1836, p. 82-84, pl. 9, fig. 1-8. - Lernæopoda stellata Rathke, 1839, p. 154-165. - Tracheliastes stellifer Neresheimer, 1909, p. 3-4, pl. I, fig. 4. - Tracheliastes stellifer Neresheimer, $1909 a$, p. 80, fig. 334.

Localités. - a. 1 sur un Silurus glanis L. long de $2 \mathrm{~m} .10$ et pesant $66 \mathrm{~kg}$., à la base du premier are branchial, Vayani [Vajani], rit. Laboretz [Laborec], 9-IV-1926. 
b. $1 \subsetneq$ sur Silurus glanis L., Tchop [Čop, Czop].

Remarques. - Le Tracheliastes de la Russie sous-carpathique est manifestement le même que Kollar trouvait en 1834-35 sur les Silures du marché de Vienne. Bien que nos exemplaires soient l'un et l'autre privés de leur bulla étoilée, leur identité spécifique ne fait aucun doute.

Wilson (1915, p. 654) écrit : "Lerneopoda stellata Blainville, 1823 , p. 112 , was figured by Milne-Edwards, 1840 , pl. 40 , fig. 12 ” et ajoute que l'espèce est trop sommairement décrite pour pouvoir

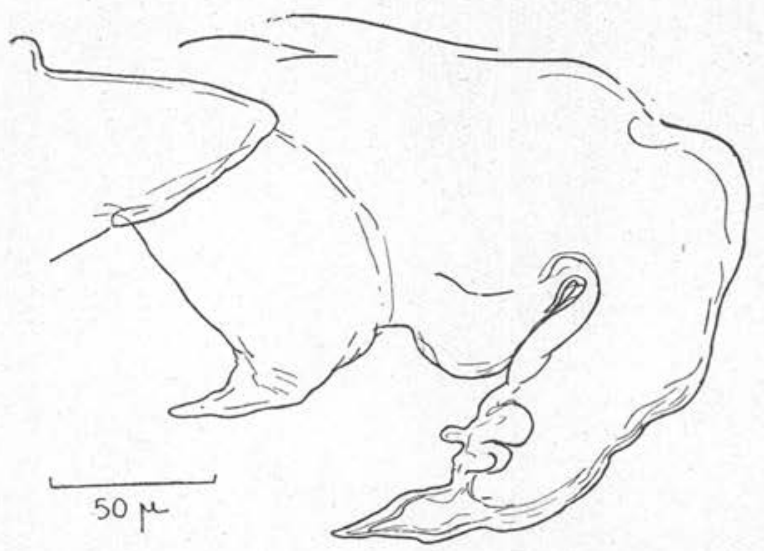

Fıg. 6. - Tracheliasles stellatus (Mayor) 우 (b). Maxillipèdes.

être reconnue. En réalité, l'espèce a été décrite seulement en 1824 par Mayor dont Milne-Edwards n'a fait que copier la figure. Il paraît hors de doute que Lerneopoda stellata Mayor, 1824, et Tracheliastes stellifer Kollar, 1836, sont une seule et même espèce : la comparaison des descriptions originales suffirait à l'indiquer, mais nous avons examiné dans les collections du Muséum deux Tracheliastes stellatus (Mayor) (avec une bulla!) sans étiquette qui sont vraisemblablement les échantillons typiques de Mayor qui en 1840 (H. Milne-Edwards, p. 515) étaient présents dans la collection ; ces échantillons sont absolument semblables à nos spécimens souscarpathiques.

Mayor donne comme hôte "le sterlet " et comme localité la Norvège. Si la localité est exacte "sterlet " est ici simplement synonyme d'esturgeon et ne désigne certainement pas le vrai sterlet (Accipenser ruthenus L.) d'Europe orientale. 
Kollar (1836) a trouvé ses spécimens sur Silurus glanis L. (marché de Vienne), et Rathke rappelle en 1839 les captures qu'il a effectuées à Iéni-Kalé, sur la rive occidentale européenne du "Bosphorus Cimmericus " (détroit de Kertch), sur la peau " des Hausen und anderer Störarten " (1839, p. 155), done sur Huso huso (L.) et d'autres espèces d'esturgeons. Enfin Neresheimer (1909) signale l'espèce en Bavière, sur Silurus glanis L.

C'est done une espèce rare qui parasite des poissons de forte taille, Accipenseridæ et Silurus glanis L., dans la Mer Noire et les

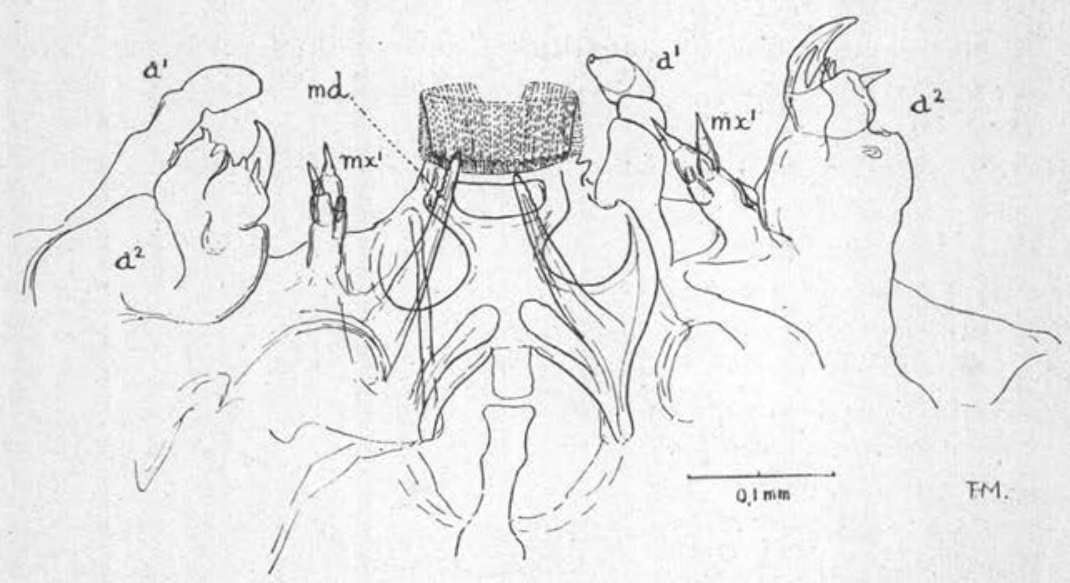

Fı́. 7. - Tracheliastes stellatus (Mayor) ㅇ (b). - Région céphalique.

bassins qui en dépendent (Danube au moins jusqu'en Bavière) (et peut-être (?) la Norvège) (1).

Le $T$. stellatus est très différent du $T$. polycolpus et présente des caractères qui font de lui le représentant d'une section spéciale du genre Tracheliastes. Ici, en effet, le céphalothorax n'est pas allongé et cylindroïde ; il est, au contraire, court et ovoïde ; les " épaules " (origine des " bras ") sont bien marquées, et le tronc est plus ou moins distinctement segmenté. Sur l'exemplaire monté nous avons observé, sur la face ventrale du trone, une série d'invaginations paires, en culs-de-sac, bien marquées par les corps étrangers qui en occupaient les récessus et qu'un examen superficiel ferait aisément prendre pour du pigment.

(1) Les seules captures certaines citées sont celles de Mayor, Rathke, Kollar et Neresheimer ; les autres références (par exemple Fatio, 1890, Faune Vert. Suisse, $\mathrm{V}$, Poissons, p. 446) paraissent n'être que des allusions à l'existence de l'espèce sur - le silure en général plutôt que la mention d'une capture précise. 
Le corps se termine par une région conique que 3 paires de sillons latéraux divisent en 3 articles - ou apparences d'articles et qui porte à son extrémité un lobe dorso-médian portant lui-même un petit mucron ventral. Wilson (1915, p. 644-645), après avoir refusé aux Tracheliastes un processus génital (p. 644), en attribue un à $T$. maculatus et à $T$. stellifer (p. 645). En réalité, la région segmentée paraît bien représenter un abdomen rudimentaire : Kollar spécifie en effet (1836, p. 84) que l'anus se trouve à son extrémité ; par contre, le petit lobe est peut-être le processus génital des autres Lerneopodidæ.

Ajoutons enfin que les maxillules sont distinctement articulées alors que Wilson écrit, à propos de la famille des Lerneopodidæ : "There is no genus in which the maxillæ [first maxillæ] show definite segmentation but they always appear one-jointed " (1915, p. 580 ).

Pour tenir compte de ces faits il apparaît nécessaire de modifier légèrement les diagnoses des Tracheliastinæ et de Tracheliastes telles qu'elles sont établies par Wilson (1915, p. 644), pour qu'elles puissent s'appliquer aussi au $T$. stellatus comme le pouvait l'excellente diagnose générique de Kollar (1836, p. 82).

Tracheliastinæ Wilson, 1915. - Femelle : céphalothorax en général plus court que les maxilles (" bras ") (parfois légèrement plus long), très étroit, allongé et subcylindrique ou court, cordiforme-ovoïde, toujours dans le prolongement du trone ou un peu incliné dorsalement (1) ; tronc allongé, subcylindrique ou plus ou moins aplati dorso-ventralement, plus ou moins élargi à son extrémité caudale (qui peut être simple ou plurilobée), généralement sans segmentation vraie apparente; pas de processus postérieurs, de lamelles anales ni de processus génital (?), mais parfois un rudiment d'abdomen ; maxillipèdes réduits, placés entre les bases des maxilles (" bras ») (2) qui sont toujours plus ou moins éloignées de la bouche et unies seulement à leur extrémité ; bulla en forme de champignon ou d'étoile. - Mâle : inconnu.

Tracheliastes von Nordmann, 1832. - Femelle : céphalothorax séparé du trone par un sillon bien marqué; somite maxillomaxillipédique bien distinct de la partie antérieure du céphalo-

(1) T. grandis Wilson $(1915$, p. $645, \mathrm{pl} .40$, fig. 106) a le céphalothorax courbé ventralement mais comme on ne connait qu'un exemplaire intact de cette espèce, la courbure ventrale de son céphalothorax pourrait bien n'être qu'un caractère individuel ; dans le cas contraire, il faudrait ajouter ici : « ou ventralement ».

(2) "On the inner surface of the second maxillae " (Wilson, 1915, p. 644) est très compréhensible et n'aura induit aucun carcinologiste en erreur mais n'en est pas moins, pris mot à mot, in exact : les maxillipèdes sont évidemment insérées sur la paroi du corps, non sur celle d'un autre appendice. 
thorax et formant des " épaules " saillantes ; pas de carapace dorsale ; antennules indistinctement articulées ; antennes biramées, la branche ventrale (externe) bi-articulée, uncifère, la branche dorsale (interne) uni-articulée (1), inerme ou faiblement spinifère; maxillule articulée ou non, bi ou tripartite; maxilles (" bras ") longues, fortes, grêles, unies seulement à leur extrémité distale, à la base d'une bulla en forme de champignon ou d'étoile; maxillipèdes réduits, à griffe faible ; cordons ovigères longs, cylindriques, variant d'une longueur inférieure à celle du tronc à une longueur supérieure à celle du corps ; taille moyenne et grande : $6-35 \mathrm{~mm}$.

On connaît actuellement cinq espèces de Tracheliastes : T. stellatus (Mayor, 1824) ; T. polycolpus von Nordmann, 1832 ; T. maculatus Kollar, 1836 ; T. gigas Richiardi, 1881 , et $T$. grandis Wilson, 1915.

Le $T$. polycolpus comprend plusieurs variétés, encore peu connues d'ailleurs. On ignore malheureusement d'où provenaient les exem-

(1) Il est extrêmement difficile d'arriver à une connaissance satisfaisante de l'antenne des Lernaopodida, ces appendices étant petits et, surtout, indistinctement segmentés. D'auıre part, il s'agit de savoir laquelle des deux branches sera considérée comme endopudiı, et laquelle sera l'exopodite. Or, l'étude attentive des travaux d'un observateur aussi consciencieux et aussi avesti que Wilson montre que sa nomenclature antennaire est loin d'être stable. La diagnose du genre Salmincola contient la phrase "both rami one-jointed " (1915, p. 603) mais la description de $S$. edwardsi mentionne une antenne avec \& the exopod rounded and unsegmented... the endopod two-jointed ) (1915, p. 610) [Cf. aussi la description de S. siscowet : " exopod one-jointed... endopod two-jointed », ibid. p. 608-609]. Notons qu'ici la branche dorsale-interne est considérée comme l'exopodite, la branche ventrale-uncifère comme l'endopodite. Il n'en est pas de même pour Achteres (p. 617 " endopod one-jointed, exopod two-jointed "). Mais voici que pour Charopinus on nous décrit t'antenne " with a curved exopod and a jointed endopod $)(1911$, p. 653$)$; or la branche articulée de l'antenne de Charopinus (pl. 42, fig. 120 [ ( endopod $»$, p. 653]) est homologue de la branche articulée de l'antenne d'Achteres ( $\mathrm{pl}$. 38. fig. 95 [ [ exopod ", p. 617]). De plus, à propos de Charopinus bicaudatus, l'auteur écrit : " both rami unsegmented, the endopod considerably larger than the exopod "' alors que la figure (pl. 42, fig. 120) représente une des branches (la ventrale-uncifère) bi-articulée, et, alors que la diagnose génerique désigne la rame développée (dorsale-inerme) comme l'exopodite et la rame réduite (ventrale-uncifère) comme l'endopodite (1915, p. 653), la diagnose spécifique emploie une terminologie exactement inverse.

Il semblerait que l'étude du développement dût éclaircir la question, d'autant plus que Wilson lui-même a consacré un excellent mémoire au développement d'Achteres ambloplites Kellicott. Le metanauplius (1911, p. 202-203. pl. 31, fig. 16-17) a, à l'antenne, un exopodite 5-articulé, un endopodite 2 -articulé avec une griffe visible sous la cuticule. Le premier stade copépodite (1911, p. 209, pl. 32, fig. 22) a un exopodite 1-articulé, un endopodite 2-articulé surmonté d'une griffe. Jusqu'ici tout est clair et l'on s'attend à voir la rame ventrale-uncifère conserver son titre d'endopodite ; mais le deuxième stade copépodite (1911, p. 213, pl. 33, fig. 30) a l'exopodite [= endopodite des stades précédents] " indistinctly two-jointed and ends in a stout curved claw $)$ tandis que l'endopodite $[=$ exopodite des stades précédents] est «. one-jointed and tipped with two minute spines ». Alors que Wilson (1915, p. 577) et d'autres (Leigh-Sharp, Messjiatzeff, etc.) considèrent la rame ventrale-externe-uncifère comme l'exopodite et la rame dorsale-interneinerme comme l'endopodite, nous préférons, pour l'instant, avec Baumann (1913, p. 162) parler, sans préjuger de leur nature, de rame externe (ventrale) et de rame interne (dorsale). 
plaires décrits par von Nordmann : nos spécimens paraissent si voisins de ceux de von Nordmann qu'on peut les considérer comme représentant la forme typique de l'espèce. La variété phoxini Vejdovsky, 1877, ne semble différer de la forme typique que par le détail morphologique des antennes: elle provient du bassin de l'Elbe, tandis que nos spécimens (et peut-être ceux de von Nordmann) ont été recueillis dans le bassin du Danube. La variété
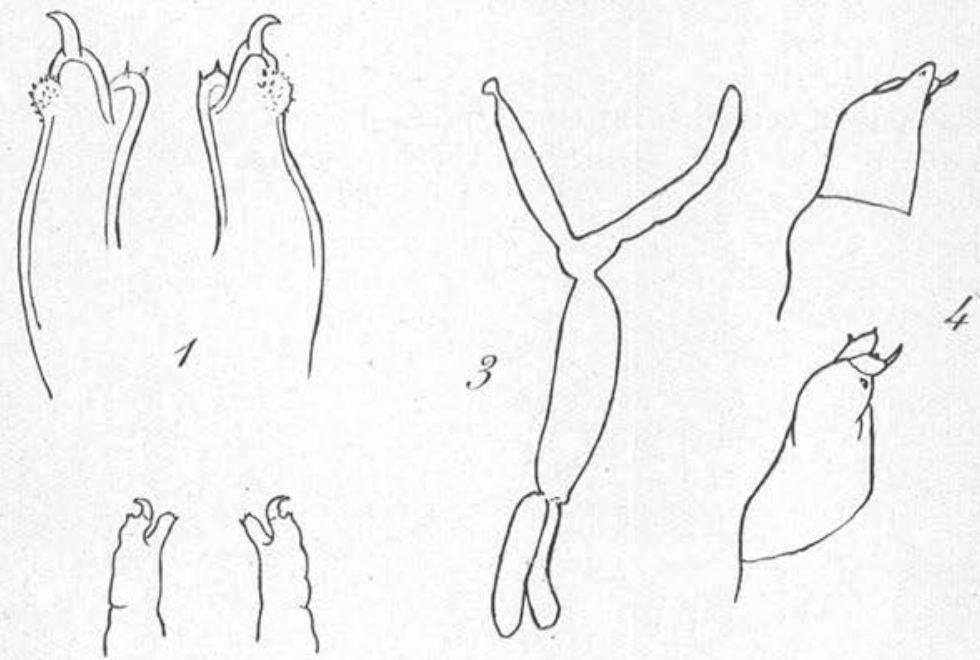

2

Fig. 8. - Tracheliastes polycolpus von Nordmann Q. $_{\text {- }}$ 1. Antennes d'après Vejdovsky (1877, pl. 3, fig. 2). - 2. Id. d'après von Nordmann (1832, pl. 7 , fig. $3(b)$. - 3. Var. baicalensis, aspect général, schématisé d'après Messjatzeff (1926, fig. 20). -4. Var. baicalensis, antenne, d'après Messjatzeff (1926, fig. 21 et 22).

baicalensis Messjatzeff, 1926, paraît caractérisée par la morphologie de ses antennes [ungulus grêle, peu recourbé, coussinet spinifère immédiatement inférieur à l'ungulus à peine saillant, rame dorsale portant « drei kleinen Zacken 》 (Messjatzeff, 1926, p. 133) (1)] et par l'élongation considérable du céphalothorax, nettement plus long que les bras. Enfin la variété nordique kessleri Messjatzeff, 1926 (p. 134) aurait pour caractéristique la présence d'une pointe simple à la mandibule, dont l'apex serait, chez les autres variétés, bifide. Il faut avouer que nous avons encore très peu de documents sur les formes des Tracheliastes européens qu'il vaudrait la peine

(1) Sans doute disposé de façon à ce qu'il soit impossible de les apercevoir tous les trois simultanément puisque la fig. 21 en montre un et la fig. 22 deux. 
de rechercher systématiquement : le $T$. maculatus Kollar de Vienne mériterait tout spécialement d'être redécrit avec soin.

\section{Basanistes huchonis (Schrank, 1786)}

Lernæa huchonis Schrank, 1786, p. 99, pl. 1, fig. A-D [fide auctorum ! Je n'ai pas vu le mémoire de Schrank cité tantôt "Baiersche Reise, 1796 " (von Nordmann, 1832, p. 87), tantôt "Voyage en
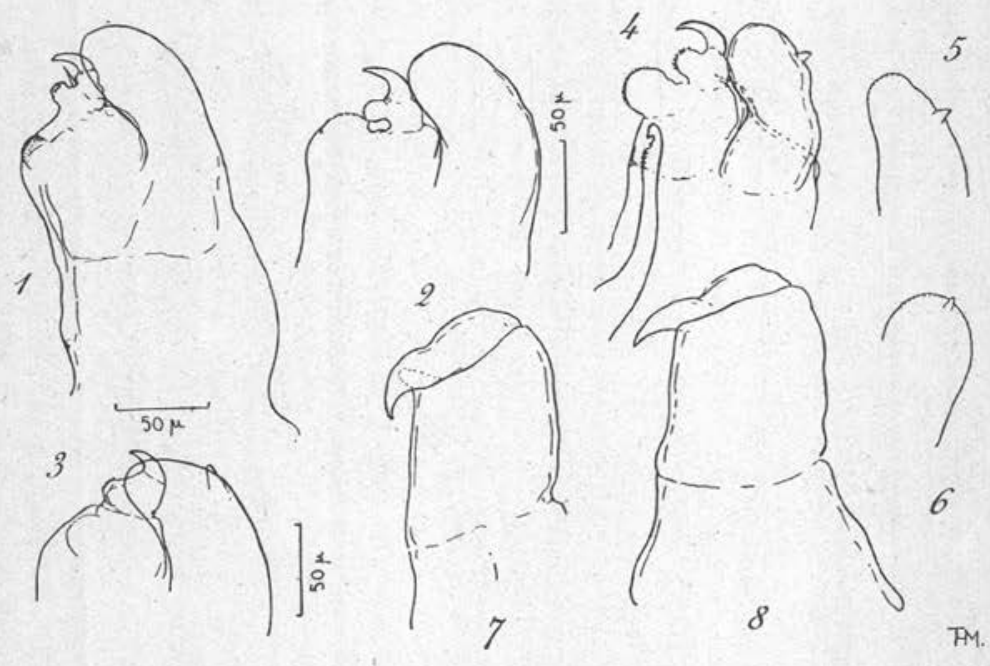

Fig. 9. - Tracheliastes polycolpus von Nordmann . . - 1. Antenne (a). - 2. Id . (b). - 3. Id. (b). - 4. Id. et mandibule, juv. (c). - 5-6. Id., extremité de la branche interne, dorsale (c). - 7-8. Maxillipèdes, juv. (c).

Bohême, 1786 " (Wilson, 1915, p. 720), tantòt "Baierische Reise, 1786 »[Lernæa huchonis, pl. I, en face p. 23] (Leigh-Sharpe, 1928, p. 184), tantôt Iter bavaricum, tantôt comme publié à Nuremberg et tantôt à Münich]. - Basanistes huchonis von Nordmann, 1832, p. 87-90. - Kollar, 1836, p. 86-90, pl. 10. - Neresheimer, $1909 a$, p. 81-82, fig. 339-340. - Leigh-Sharpe, 1928, p. 183-184, fig. 4.

Localités. - $-a .15$ ᄋ, à la face interne de l'opercule de Hucho hucho (L.), Ganitchi [Ganiči], riv. Teresva (affl. de Tisa), 23-VIII1 c24.

b. 12 q, ibidem, Podplecha [Podpleša], riv. Teresva (affl. de Tisa), 27-IV-1924.

c. 34 q, ibidem, Krașnochora [Krasnošora], riv. Teresva (affl, de Tisa), 1927. 
d. 8 , , ibidem, eodem loco.

e. 3 f, ibidem, Ganitchi [Ganiči], riv. Teresva (affl. Tisa)

Distribution. - Europe centrale (Bassin du Danube).

Observations éthologiques. - Forme très fréquente pour la région envisagée (1) mais ne se rencontrant que sur les spécimens d'une certaine taille (d'au moins 3 ans et $30-40 \mathrm{~cm}$. de longueur).
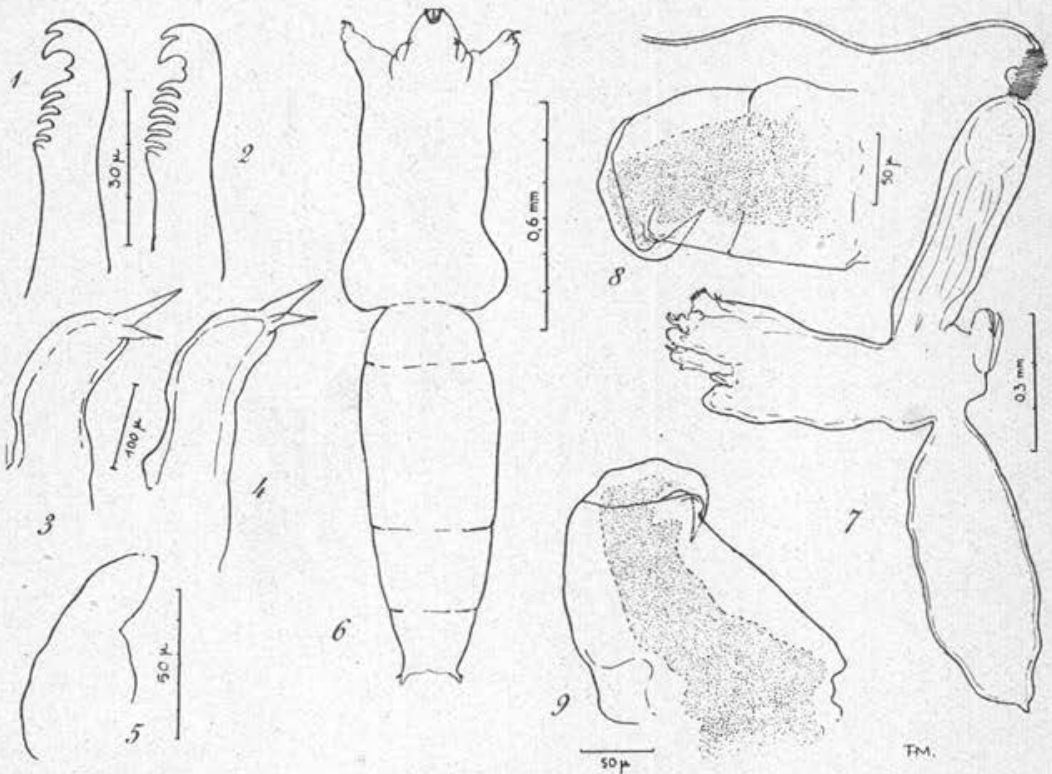

FIG. 10. - Tracheliastes polycolpus von Nordmann Q. - 1-2. Extrêmité de la mandibule, (e). - 3-4. Maxillules, juv. (c). - 5. Antennule, juv. (c). - 6. Aspect ventral juv. (c). - 7. Aspect latéral, juv. (d). - 8-9. Maxillipèdes de l'exemplaire juv. (d).

Les alevins et les jeunes paraissent toujours indemnes ; ils habitent d'ailleurs des endroits peu profonds, dans la zone d'inondation du fleuve et non dans le lit mineur de ce dernier. Ils restent là pendant 1 an $1 / 2$ à 2 ans pour commencer ensuite à descendre jusqu'à l'embouchure des affluents principaux, dans un secteur où l'eau est moins aérée. C'est à partir de ce stade qu'on les trouve parasités. Les Basanistes paraissent plus abondants au printemps, à l'époque du frai (mars-avril). Parfois toute la paroi interne de l'opercule est

(1) Comme pour le genre Tracheliastes le $\sigma^{\top}$ de Basanistes est inconnu. On ne saurait trop souhaiter que les zoologistes danubiens se préoccupent de la question. 
couverte de Copépodes, qui occupent alors une surface pouvant atteindre environ $30 \mathrm{~mm}$. de diamètre. Les pêcheurs racontent que le poisson, pour se débarrasser de son parasite, recherche des endroits à courant violent, et ouvre la bouche dans ces rapides pour la faire nettoyer.... En ruthène, le parasite se nomme " vouchi » [vuši], c'est-à-dire "pou ".

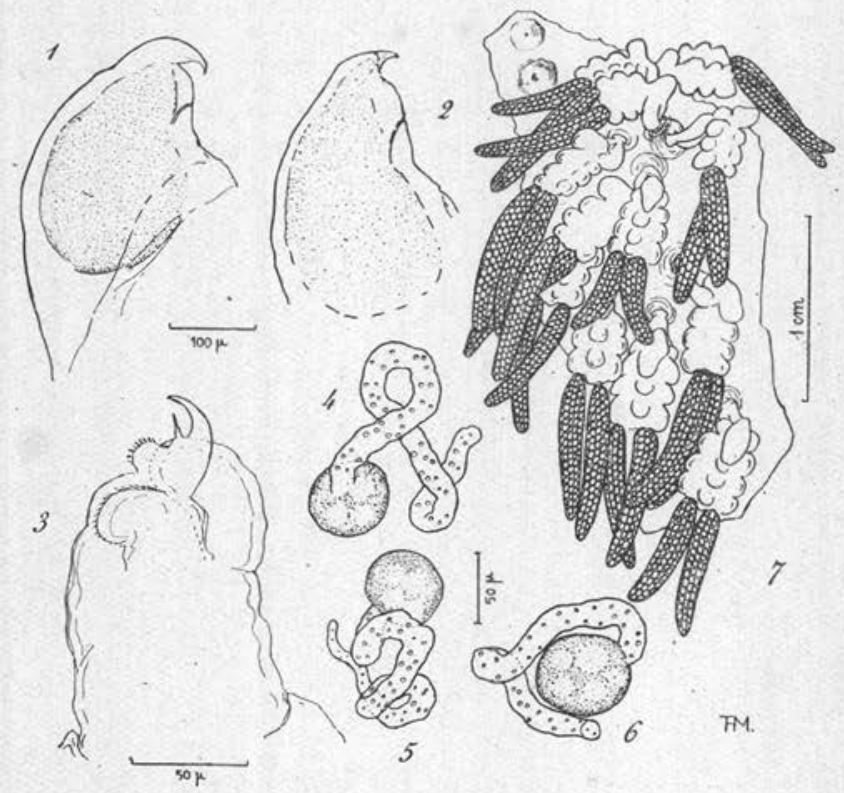

FIg. 11. - 1-2 Tracheliasles polyçolpus von Nordmann $ᄋ$, maxillipèdes d'un exemplaire de $7 \mathrm{~mm}$. de long $(a) .-3$. Id., antennes, iuv. (c). $-4-6$. Id., trois oocytes avec leur filament accessoire (limites cellulaires non figurées) $(b) .-7$. Basanistes huchonis (Schrank) $ᄋ$, un groupe (c) in situ à la face interne de l'opercule de Hucho hucho (L.).

\section{Liste de Copépodes parasites et de Branchiures DULCaQuicoles D'Europe (incl. Islande et Grœnland)}

Comme le titre précédent l'indique assez, il ne s'agit pas ici d'un inventaire complet et critique, mais d'une simple liste de Copépodes parasites et de branchiures, notés au cours des recherches bibliographiques nécessitées par le présent travail. Cette liste est certainement incomplète, mais, si imparfaite qu'elle soit, elle peut rendre quelques services et peut donner matière à des remarques d'ordre général. Elle n'est pas critique en ce sens qu'il ne s'agit que 
d'une compilation des données, plus ou moins exactes, fournies par la littérature.

\section{COPÉPODES}

\section{Ergasilidæ}

1. Ergasilus sieboldi von Nordmann. - Perca fluviatilis L., Acerina cernua (L.), Silurus glanis L., Esox lucius L., Cottus gobio L., Pleuronectes flesus L., Cyprinus carpio L., Carassius carassius (L.), Tinca tinca (L.), Barbus barbus (L.), Gobio gobio (L.), G. g. carpathicus Vladykov, Abramis brama (L.), Blicca björkna (L.), Vimba vimba (L.), Alburnus alburnus (L.), Leuciscus cephalus L., L. leuciscus (L.), L. idus (L.), Scardinius erythrophthalmus (L.), Rutilus rutilus (L.), Anguilla anguilla L. (1).

2. Ergasilus gibbus von Nordmann, 1832. - Anguilla anguilla (L.), Rutilus rutilus (L.) (?).

3. Ergasilus trisetaceus von Nordmann, 1832. - Acerina cernua (L.), Silurus glanis L., Tinca tinca (L.).

4. Ergasilus hoferi Borodine, 1915. - Abramis brama (L.) (2).

5. Ergasilus surbecki Baumann, 1912. -- Coregonus wartmanni (Bloch), C. schinzi palea Cuvier, Esox lucius L.

6. Thersitina gasterostei Pagenstecher, 1861. - Gasierosteus aculeatus L., Pygosteus pungitius (L.), Spinachia vulgaris Flem.

\section{Caligidæ}

7. Galigus lacustris Steenstrup et Lütken, 1861. - Perca fluviatilis L., Esox lucius L., Gasterosteus aculeatus L. Pygosteus pungitius (L.), Blicca björkna (L.), Phoxinus phoxinus (L.), Leuciscus idus (L.), Rutilus rutilus (L.).

8. Lepeophtheirus thompsoni Baird, 1850. - Lucioperca lucioperca (L.) (fide Gadd. 1904, sub, nom. « L. branchialis Malm apud. Steenstrup et Lütken, $1861 »)$.

9. Lepeophtheirus salmonis (Kröyer, 1837). - Salmo salar L., S. trutta L.

10. Lepeophtheirus sturionis (KKröyer, 1837). - Accipenser sturio $\mathrm{L}$.

(1) Les Ergasilus sielboldi signalés sur Coregonus lavarelus (L.), C. wartmanni nobilis Fatio, C. exiguus albellus Fatio, C. schinzi helveticus Fatio, Salvelinus salvelinus (L.), Trutta lacustris (L.), n'appartiennent probablement pas à cette espèce.

-(2) Borodine donne Abramis comme l'hôte de son E. hoferi et Pesta, 1928 donne la "Schleie " c'est-à-dire Tinca tinca (L.). 


\section{Dichelesthiidæ}

11. Lamproglena pulchella von Nordmann, 1832. - Leuciscus cephalus (L.) (incl. cavedanus Bonap.), L. leuciscus (L.), L. idus (L.), L. agassizi (Heckel), Scardinius erythrophthalmus (L.), Alburnus alborella De Filippi, Aspius rapax (L.).

12. Dichelesthium oblongum (Abildgaard, 1794) (= stùrionis Hermann, 1804). - Accipenser sturio L.

\section{Chondracanthidæ}

pro memoria

Je ne sais pas trop pourquoi on compte parfois Diocus gobinus (O.-F. Müller, 1776) au nombre des Copépodes parasites d'eau douce européens : c'est ainsi qu'il figure dans les Copépodes parasites de la Süsswasserfauna (Neresheimer, 1909 a). En réalité, il s'agit d'une espèce groënlandaise (Groënland occidental) et marine, parasite de Phobetor ventralis C. V. (= tricuspis Reinh., = Cottus gobio auctorum veterum nonnullorum nec C. gobio L.) (cf. Hansen, 1923, p. 48-49). C'est probablement le nom donné à l'hôte par certains auteurs anciens (y compris le descripteur de l'espèce) qui est à l'origine d'une semblable confusion.

\section{Lerneopodidæ}

13. Salmincola carpionis Kröyer, 1837. - Salvelinus alpinus L.

14. Salmincola lotæ Olsson, 1877. - Lota lota (L.).

15. Salmincola salmonea (Gissler, 1751). - Salmo salar L., S. trutta L.

16. Salmincola alpina Olsson, 1877. - Salvelinus alpinus (L.), Gasterosteus aculeatus L. var. dimidiata.

17. Salmincola edwardsi (Olsson, 1869). - Salvelinus salvelinus (L.), S. fontinalis Mitch., Coregonus maræna (Bloch).

18. Salmincola extumescens Gadd, 1901. - Coregonus lavaretus (L.),

19. Salmincola coregonorum-(Kessler, 1868).-- Coregonus lavaretus (L.), C. fera Kessler, C. widegreni Malm.

20. Salmincola thymalli (Kessler, 1868). - Thymallus thymallus (L.), Salvelinus alpinus (L.), Coregonus lavaretus (L.).

21. Salmincola extensa (Kessler, 1868). - Coregonus widegreni Malm.

22. Salmincola heintzi Neresheimer, 1909. - Salvelinus salvelinus (L.). 
23. Achteres percarum von Nordmann, 1832. - Perca fluviatilis typica et vulgaris Schæffer, Lucioperca volgensis (Gmelin), Esox lucius $\mathrm{L}$.

24. Achteres sandræ Gadd, 1901. - Lucioperca lucioperca (L.).

25. Achteres coregoni Baumann, 1912. - Coregonus wartmanni alpinus Fatio.

26. Clavellisa emarginata (Kröyer, 1837). - Alosa fallax Lac. (=- finta Cuv. et auct.), A. alosa (L.).

27. Tracheliastes polycolpus von Nordmann, 1832. - Leuciscus idus (L.), Phoxinus phoxinus (L.), Chondrostoma nasus (L.), Rutilus rutilus lacustris (Pallas) (lac Baïkal), Barbus barbus (L.), B. meridionalis petenyi Heckel, Abramis brama (L.).

28. Tracheliastes maculatus Kollar, 1835. - Abramis brama (L.), Blicca björkna (L.).

29. Tracheliastes stellatus (Mayor, 1824). - Silurus glanis L., Accipenser sturio L., Accipenser spp.

30. Basanistes huchonis (Schrank, 1786). - Hucho hucho (L.).

31. Basanistes nordmanni Kessler, 1868. - Stenodus leucichthys (Güld.).

32. Basanistes coregoni Neresheimer, 1909. - Coregonus wartmanni (Bloch), C. wartmanni compactus Fatio, C. macrophthalmus Nüsslin, C. acronius Rapp.

\section{Lernaeidæ}

33. Lernæa esocina (Bürmeister, 1835). — Esox lucius L., Lota lota (L.), Cottus gobio L., Perca fluviatilis L., Gasterosteus aculeatus L., Cobitis tænia L., Carassius carassius (L.), Tinca tinca (L.), Phoxinus phoxinus (L.), Abramis brama (L.), Gobius fluviatilis C. V.

34. Lernæa cyprinacea L., 1758. --- Carassius carassius (L.). Le L. cyprinacea, signalé au Japon sur Carassius auratus (L.), des tritons, Diemictylus pyrrhogaster (Boie) et des têtards de Rana catesbiana Shaw dans Dobutsugaku Zasshi, XXVII, 1915, p. 459, et par Yô K. Okada (cf. Yô K. Okada, Copépode parasite des Amphibiens, A nnot. Zool. Jap., 11, n², 1927, p. 185-187) semble identique au L. elegans Leigh-Sharpe, 1925 (1) qui a été recueilli, au Japon, sur Cyprinus carpio L., Carassius auratus (L.), Pseudorasbora anguillicaudata (Cantor) et Anguilla japonica Schlegel.

(1) Cf. aussi (cit. fide Zool. Rec.) : N. Nakai, On the development of a parasitic Copepod, Lernaa elegans Leigh Sharpe, infesting Cyprinus carpio L. (Journ. Imp. Fish. Inst. Tokyo, XXIII, 1927, p. 39-59, 7 fig. texte, 3 pls.) et T. Matsui et Kumada, "Ikari-Mushi " (Lerncea elegans Leigh-Sharpe), a new parasitic copepod of japanese eel (ibid. ; XXIII, 1928, p. 101-107, 3 pl.). 
35. Lernæa phoxinacea (Kröyer, 1863). - Phoxinus phoxinus (L.), Cyprinus carpio L.

\section{BRANCHIURES}

\section{Argulidæ}

36. Argulus foliaceus L. - Trutta irideus var. shasta Jordan, Salmo trutta typica et fario (L.), Gasterosteus aculeatus L., Esox lucius L., Perca fluviatilis L., Lucioperca lucioperca (L.), Acerina cernua (L.), Pleuronectes flesus L., Syngnathus typhle L. (Baltique), Cyprinus carpio L.; Tinca tinca (L.), Abramis brama (L.), Blicca björkna (L.), Leuciscus idus (L.), L. cephalus L., Phoxinus phoxinus (L.), Rutilus rutilus (L.), Alburnus alburnus.(L.), Rhodeus sericeus (Pallas), têtards de grenouilles et de crapauds.

37. Argulus coregoni Thorell, 1864 (incl. A. phoxini Leydig, 1871, cf. von Nettovich, 1902, p. 2). - Coregonus lavaretus (L.), Thymallus thymallus (L.), Salmo trutta L., Leuciscus idus (L.), Esox lucius L., Lampetra planeri (Bloch).

38. Argulus viridis von Nettovich, 1902. - Cyprinus carpio L., Phoxinus phoxinus (L.), Alburnus alburnus (L.), Rhodeus sericeus (Pallas).

39. Argulus matritensis Arévalo, 1921. - Hòte non indiqué, le type ayant été recueilli libre.

Je saisis l'occasion pour signaler, après Steuer (1928), l'état déplorable dans lequel se trouve notre connaissance de la systématique des Argulidés d'Europe : le fait est que l'on ignore aujourd'hui combien il y a d'espèces d'Argulus en Europe et quels sont leurs caractères spécifiques. Les groupes demeurés monotypes pendant un siècle et très souvent cités sont en effet exposés à de semblables mésaventures de par le caractère respecté de la tradition dont ils deviennent l'objet et les habitudes paresseuses que cette dernière favorise.

Steuer a signalé quelques-unes des incertitudes de la littérature. Ajoutons seulement pour l'instant que l'Argulus foliaceus L. de Th. et A. Scott (1913, p. 227-228, pl. LXXII, fig. 1-10) n'est pas l'espèce européenne commune: l'exemplaire figuré par les Scott est vert foncé (cf. A. viridis von Nettovich !), à carapace orbiculaire et à lobes abdominaux aigus ; les caractères sexuels des pattes natatoires ô ne sont pas identiques. Les figures 8-10 (et peut-être toute la planche) attribuées à une $q$ concernent en réalité un $\delta$. 


\section{Remarques}

Les Copépodes parasites des eaux douces d'Europe sont encore très mal connus : très souvent cités ils le sont encore trop souvent comme si tous les Ergasilus étaient E. sieboldi, tous les Achteres, A. percarum et tous les Argulus, A. foliaceus.

Il n'est donc nullement certain que tous les poissons donnés par les auteurs pour des hôtes d'Ergasilus sieboldi, par exemple, aient été effectivement porteurs de cette espèce, et non pas d'un autre Ergasilus. Les données précises sur la distribution géographique des espèces font à peu près défaut et ne seront acquises que lorsque la position systématique de celles-ci sera solidement établie. Or ce n'est pas toujours le cas : les Salmincola, les Achteres, les Tracheliastes, les Argulus, etc., devront être étudiés en détail, sur un matériel abondant de provenance certaine (hôte, localité, date, altitude, etc.).

Dans quelques cas, on constate une localisation spécifique d'un parasite sur un hôte déterminé; cette spécificité peut n'être qu'apparente si la monoxénie en question ne tient qu'à l'insuffisance actuelle de nos connaissances, comme c'est peut-être le cas pour Ergasilus hoferi Borodine, qui n'est connu que d'Abramis brama (L.), pour nombre de Salmonicola, etc. Ailleurs cette spécificité peut être authentique et constante, par exemple pour Dichelestium oblongum (Abildgaard) et Lepeophtheirus sturionis (Kröyer) qui semblent ne parasiter que l'Accipenser sturio L., ou pour Basanistes huchonis (Schrank) qu'on n'a jamais trouvé ailleurs que sur Hucho hucho (L.).

Ailleurs, la liaison du parasite n'est plus avec un seul hôte déterminé mais avec un groupe d'hôtes, ce groupe étant ou non systématique (composé de genres ou d'espèces affines), mais en tous les cas toujours biologique (composé de genres ou d'espèces ayant en commun des caractères écologiques ou chorologiques identiques ou similaires). Le Lamproglena pulchella von Nordmann n'a été trouvé jusqu'ici que sur des poissons (Cyprinidés) d'eaux à courant peu rapide, les Salmincola que sur des poissons d'eaux froides ou aérées. Les Lernæa, au contraire, et le Caligus lacustris S. et L. sur des poissons eurythermes. Souvent, à l'intérieur d'un genre, s'opposent deux ou plusieurs espèces, les unes caractéristiques de poissons eurythermes, les autres de poissons sténothermes d'eaux froides ; c'est ainsi qu'on a, en face d'Ergasilus sieboldi von Nordmann : E. surbecki Baumann (Corégones); en face de Basanistes huchonis (Schrank) : B. nordmanni Kessler et B. coregoni Neresheimer 
(Corégones) ; en face d'Achteres percarum von Nordmann : A. coregoni Baumann ; en face d'Argulus foliaceus L. : A. coregoni Thorell.

Tout ceci est encore peu apparent parce qu'on manque de renseignements précis et qu'on n'est pas toujours sùr quand un auteur cite Ergasilus sieboldi, Achteres percarum ou Argulus foliaceus qu'il s'agit d'autre chose que d'une détermination simplement générique. Cependant on entrevoit, pour l'avenir, dans une comparaison détaillée de spécimens abondants provenant de régions différentes (1), séparées par des distances verticales ou horizontales (donc soit de bassins différents, soit des diverses altitudes d'un même bassin), le principe d'une étude plus satisfaisante des Copépodes parasites dulcaquicoles paléarctiques. Seulement, il faut pour cela un matériel suffisant, qui ne sera jamais trop considérable, ce qui implique la récolte systématique des parasites en des points aussi nombreux que possible, c'est-à-dire un travail long, obscur et difficile.

Addendum. - Un travail tout récent de E. Neuhaus (Untersuchungen über die Lebensweise von Ergasilus sieboldi Nord., Zeitschr. für Fisch., XXVII, 3, 1929, p. 339-397, 26 fig., 7 tableaux, $2 \mathrm{pl}$.) conclut à l'identité spécifique d'E. trisetaceus, surbecki, hoferi et sieboldi.

\section{BiBLIOGRAPHiE}

Arévalo (C.). - Uu nuevo Argulus español. Bol. Real Soc. Esp. Sc. Nat., XXI, 1921, p. $108-110,1$ fig.

Baumanx (F.). - Parasitische Copepoden auf Coregonen. Ein Beilrag zur Kenntniss der parasitischen Copepoden der Schweiz. Zool Anz., XL, 1912, p. 53-57, 2 fig.

- Parasitische Copepoden auf Coregonen. Ein Beitrag zur Kenntniss der parasitischen Copepoden der Schweiz. Rev. Suisse de Zool., XXI, 1913, p. $147-178, \mathrm{pl} .5$.

Blainville (M.-H.-D.). - Mémoire sur les Lernées. Journ. de Physique, XCV, 1822 , p. $372-380$ et $437-447,1$ pl.

- Article " Lernée » in Dict. Sc. Nat., XXVI, 1823, p. 112-130.

Borodin (D.). - Ergasilus hoferi n. sp. Zeitschr. für Fish., XVII, 1915, p. 201-207, 12 fig.

Chaus (C.). - Über den Bau und die Entwickelung von Achteres percarum v. Nordm. Zeilschr. wiss. Zool., XI, 1861, p. 287-308, pl. XXIII-XXIV.

- Neue Beiträge zur Kenntniss parasitischen Copepoden. nebst Bemerkungen über das System derselben. Zeitschr. wiss. Zool., XXV, 1875, p. 327-360, pl. XXII-XXIV.

(1) Plutôt que d'hôtes différents. 
Desmarest (A.-G.). - Considéralions générales sur la classe des Crustacés, Paris, 1825 , XIX +447, p., 5 tableaux, 56 pl.

Freidenfelt (T.). - Morphologiseh Systematische Bemerkungen über Ergasilus Sieboldi Nordm., nebst vorlaüfigen Mitteilungen über die Lebensgeschichte des Tieres. Lunds Univ. Arsskr. N. F. Afd, 2, VI, 1910, n 3,12 p. 2 fig.

Frı̌̌ (A.). - Práce zoologického odděleni pro přirodovédecké proskou máni ěech, 1872,280 p., 95 fig.

Frí (A.) et VáVRA (V.). - Untersuchungen über die Fauna der Gewässer Böhmens V. Archiv. d. naturw. Landesdurchforschung von Böhmen, XI, 1901, $\mathrm{n}^{\circ} 3$, 154 p., 119 fig.

Frič (A.). - České ryby a jich cizopasnici, Prague, 1908, 80 p., 111 fig.

GadD (P.). - Parasit-Copepoder i Finland. Acta Soc. Fauna Flora Fennica, XXVI, $\mathrm{n}^{\circ} 8,1904,60$ p., 2 pl.

HoFer (B.). - Handbuch der Fischkrankheiten, München, 1904, XV, 359 p., 18 pl., 222 fig.

Kollar (V.). - Beiträge zur Kenntniss lernäenartiger Crustaceen. Ann. Wiener Museums der Naturgesch., I, 1837, p. 79-92, pl.IX-X.

Kröyer (H.). - Bidrag til Kundskab om Snyltekrebsene. Naturhist. Tidskr., (3). II, 1863 , p. 15-426, pI. 1-18.

Kunz (W.). - Studien über die Familie der Lernaeopodiden. Zeitschr. wiss. Zool., XXIX, 1877, p. 380-423, pl. XXV-XXVII.

Lehmann (C.). - Ergasilus surbecki, ein neuer Parasit für die Kleine Maräne (Coregonus albula). Schriften d. Phys.-Ökon. Ges. Königsberg, LXV, 1926, p. 61-65.

Leigh-Sharpe (W. H.). - Lernea (Lerneocera) elegans n. sp., a parasitic Copepod of Anguilla japonica. Parasitology, XVII, 1925, p. 245-251, 5 fig.

- The genera Sphyrion and Basanistes as represented by the collection in the Bristish Museum. Parasitology, XX, 1928, p. 179-184, 4 fig.

Miıor. - Notice sur une nouvelle espèce de lernéopode. Bull. Soc. Philom., février 1824, p. 24-25, pl. 1, fig. 1-8 [avril 1824].

Messjatzeff (I. I.). - Parasitische Copepoden ans dem Baikal-See. Arch. f. Nat., XCII A, 1926, p. 120-134, 23 fig.

Milne-Edwands (H.). - Histoire naturelle des Crustacés, III, 1840, 605 p. et atlas p.p.

Neresheimer (E.). - Studien über süswasser Lernäopodiden, Ber. aus $d$. Kgl. Bayer. Biol. Versuchstation, München, II, 1909, p. 1-9, pl. I et 1 fig. texte [A].

- Copepoda. 2. Teil. Die parasistischen Copepoden. Süsswasserfauna Dentschlands, $11,1909^{\text {a }}$, p. 7084 , fig. $311-345$.

Netrovich (L. von). - Neue Beiträge zur Kenntniss der Arguliden. Arb. Zool. Inst. Unib. Wien., XIII, 1902, p. 1-32, pl. I-II.

Nordann (A. von). - Micrographische Beiträge zur Naturgeschichte der wirbellosen Thiere. Heft II. Berlin, 1832, 150 p., 10 pls.

Nufer (W.). - Die Fische des Vierwaldslättersees und ihre Parasiten. Inaug. Dissert., Luzern, 1905, 232 p. 4 pl.

Pesta (O.). - Krebstiere oder Crustacea. I : Ruderfüsser oder Copepoda (1. Calanoida et 2. Cyclopoida) in Tierwelt Deutschlands, 9, 136 p., 115 fig.

Piesbergen (F.). - Die Ekto-und Endoparasiten von welchen die in der Umgebung von Tübingen lebenden Fische bewohnt werden. Jahresheft $d$. Ver. f. vaterl. Naturk. in Württemberg, 1886, p. 73-88, pl. II. 
Rathke (H.). - Zur Fauna der Krym. Mém. Acad. Imp. Sc. St. Petersbourg, Savanls étrangers, III, 1835 , p. 291-454, 10 pl.

- Zur Morphologie. Reisebemerkungen aus Taurien, 1837, 192 p., 4 pl. ; cf. Lernaopoda stellata, p. 35.

- Bemerkungen über den Bau des Dichelesthium sturionis und der Lernæopoda slellata. Nova Acta Phys.-med. Acad. Caes. Leop. Car. Nat. curiosorum, XIX, 1,1839 , p. $125-168$, pl. XVII.

Richiardi (S.). - Sopra due nuove specie di Crostacei parassiti. Zool. Anz., IV, 1881, p. 501-505 [id. (fide Brian, Copepodi parassiti dei pesci d'Italia, 1906, p. 164), Proc. verb. Soc. Tosc. Sc. Nat., Adunanza 5 luglio 1881].

Scotr (Th.) et Scotr (A.) - The British parasitic Copepoda., Ray Society, 1913, I (IX +256 p., 2 pl. $)$ et II (XI p. et 72 pl. $)$.

Schrank, (F. von Paula). Baierische Reise, München. (cf. p. 214).

Steuer (A.). Zur Systematik der Karpfenläuse. Tiroler Fischer, III, no 1, 1928, 2 p.. 2 fig.

Vesdovsky (F.). - Untersuchungen über die Anatomie und Metamorphose von Tracheliastes polycolpus Nordmann. Zeitschr. wiss. Zool., XXIX, 1877, p. 15-46, pl. 11-IV.

Voigt (M.). - Beiträge zur Kenntniss des Vorkommens von Fischparasiten in den Plöner Gewässern. Forsch. Ber. d. Biol. Station in Plön, X, 1903, p. 94-99.

Wegener (G.). - Die Ektoparasiten der Fische Ostpreussens. Aus dem Zool. Mus. der Univ. Königsberg, 1910.

Wilson (Ch. B.). - North American Copepods. - Part 9, The Lernæopodidæ, Proc. U. S. Nat. Mus., XXXIX, 1911. no 1783 (jan. 9), p. 189-226, pl. 29-36.

- North American parasitic Copepods belonging to the Lerneopodide, with a revision of the entire family. Proc. U.S. Nat. Mus., XCVII, 1915, n' 2063 , p. 565-729, 15 fig., pl. 25-56.

- North American parasitic Copepods belonging to the Lernaide, with a revision of the entire family. Proc. U.S. Nat. Mus., CIII, 1917, nº 2194 (june 13) p. 1-150, pl. 1-21.

Zivdt (I.). - Fischparasiten des Bodensees. Centralbl. für Bakt., Abt. I, Oıig. XCII, 1924, p. 225-271, 33 fig.

Laboratoire de M. le Professeur A. Gruvel, Museum national d'Histoire naturelle. 